\section{Ein Nachruf}

\author{
Am 7. Juli 2001 verstarb nach \\ schwerer Krankheit \\ Prof. Dr. med. Hellmuth \\ Kleinsorge nach einem außer- \\ gewöhnlich aktiven Leben.
}

A m 12. April 1920 in Bonn geboren, behielt Hellmuth Kleinsorge auch in schwierigen Situationen seinen rheinischen Frohsinn und Optimismus. Das Medizinstudium, durch die Kriegswirren kompliziert, konnte er 1945 mit Staatsexamen und Promotion erfolgreich abschließen. Thema der Promotionsarbeit war „Pervitin-Sucht und -Schädigungen“. Schon fünf Jahre später habilitierte er sich für das Fachgebiet Innere Medizin. Bereits mit 33 Jahren wurde er zum Professor für Innere Medizin und Direktor der Medizinischen Universitäts-Poliklinik in Jena berufen (in der Nachfolge seines Lehrers Felix Lommel). Von 1958 bis 1962 war er Dekan der Medizinischen Fakultät der Friedrich-Schiller-Universität Jena.

Der politischen Entwicklung nach 1945 stand er kritisch gegenüber. Dies führte im Zusammenhang mit dem Mauerbau zur offenen Konfrontation. Klinikdirektorat und Lehrbefugnis wurden ihm entzogen. Als Direktor der Bezirksklinik Schwerin und Chefarzt der Medizinischen Klinik sollte er sich bewähren. Doch da die politisch bedingten Querelen sich fortsetzten, entschloss er sich 1968 zur Flucht in die Bundesrepublik.

Nach einer komplizierten Übergangsperiode gelang ihm eine neue Karriere. Obgleich er bereits 1969 eine Professur am Klinikum Mannheim der Universität Heidelberg erhielt, konzentrierte sich seine Tätigkeit auf die pharmazeutische Forschung - zunächst als Forschungsleiter der Knoll AG, später in der BASF. Von 1981-87 war er Hauptgeschäftsführer der PaulMartini-Stiftung, von 1987-92 Herausgeber der „Neuen Ärztlichen“ im Rahmen der FAZ. Bis zu seinem Tode war er gesuchter Berater und Gutach-

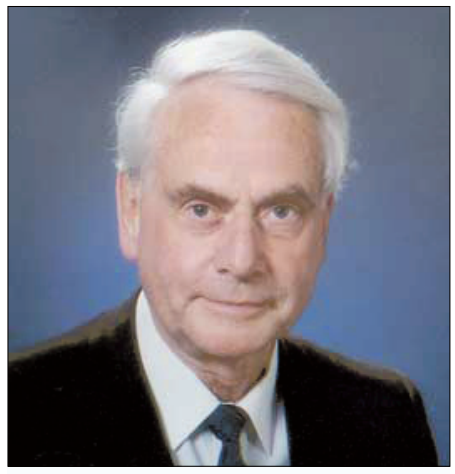

Prof. Dr. med. Hellmuth Kleinsorge

ter sowohl für pharmazeutische Unternehmen als auch staatliche Dienststellen, für wissenschaftliche Verlage wie ärztliche Gesellschaften und Vereinigungen.

Sein Interessensgebiet war außergewöhnlich breit. Ausgehend von der klassischen Inneren Medizin beschäftigte er sich vor allem mit der Psychosomatik, der klinischen Pharmakologie und - nicht zuletzt - der Allergologie und klinischen Immunologie. Bereits 1953 beschrieb er die blutdrucksenkende Wirkung des späteren Carbutamids und 1958 die antiarrhythmische Wirkung des Ajmalins.

\section{Kampf gegen}

\section{deutsch-deutsche Mauern}

Hellmuth Kleinsorge gehörte zu den Gründern des zunächst noch gesamtdeutschen Forschungsrates für AllergieFragen wie auch der zunächst noch gemeinsamen Zeitschrift „Allergie und Asthma“. Nach der Wende setzte er sich für die Zusammenführung mit der inzwischen gegründeten „Allergologie“ ein. Auch in komplizierter Situation hielt er die deutsch-deutschen Kontakte aufrecht. Nach der Wende wurde er zum letzten Ehrenmitglied der „Gesellschaft für klinische und experimentelle Immunologie der DDW“ gewählt. Die Mitte der 50er Jahre in Jena gegründete „Allergie- und Asthma-Zentrale" war die erste ihrer Art in Deutschland. In dieser entwickelten sich erste persönliche Kontakte - zunächst als Doktorand, später als Mitarbeiter. Hellmuth Kleinsorge war kein einfacher Chef. Er sprühte voller Ideen - und wollte Ergebnisse - möglichst gleich morgen. Er stimulierte Aktivitäten, war ein interessierter Begleiter und gewährte jede erdenkliche Unterstützung. Bemerkenswert war sein Gespür für neue Entwicklungen. Von seiner Kreativität zeugen etwa 400 wissenschaftliche Publikationen und 20 Buchveröffentlichungen. Seine Verdienste wurden durch zahlreiche Ehrenmitgliedschaften und andere Auszeichnungen gewürdigt.

In all dieser Zeit stand seine Frau zuverlässig an seiner Seite. Der Tod ereilte ihn zu einem Zeitpunkt, zu dem er definitiv Abschied vom aktiven und öffentlichen Leben genommen hatte eine Entscheidung, die ihm schwer gefallen war. Seine Absicht, sein außergewöhnlich reiches Leben aufzuarbeiten, blieb unvollendet.

Prof. Dr. L. Jäger, Jena

Ausschreibung -

Pulmedica-Preis 2001

Die Gesellschaft für Lungen- und Atmungsforschung verleiht jährlich den Pulmedica-Preis für Pneumologie. Dieser Preis, dotiert mit 10.000 DM wird von der Firma $3 \mathrm{M}$ Medica gestiftet. Die Ausschreibung richtet sich an alle Wissenschaftler, die sich mit Themen aus der pneumologischen Grundlagenforschung und der klinischen Pneumologie beschäftigen.

Teilnahme-Voraussetzungen:

- Deutsche oder englische Originalarbeiten, die zur Publikation vorgelegt oder angenommen, jedoch noch nicht veröffentlicht sind

- Pro Arbeitsgruppe nur eine Arbeit

- Keine anderweitige Bewerbung um einen Preis

- Autoren sollten sich nicht in leitender Stellung befinden

- Einreichung als anonymisierte, fünffache Ausfertigung beim

Sekretariat der Gesellschaft für Lungen- und Atmungsforschung in der Deutschen Gesellschaft für Pneumologie e.v.

Bürkle-de-la-Camp-Platz 1

D-44789 Bochum

Einsendeschluss ist der 10. September 2001. 